\title{
Caenorhabditis elegans nuclear hormone receptor NHR-14, cooperates with p53/cep-1 to regulate DNA damage-induced apoptosis
}

\author{
Junling Shen ${ }^{1, *}$, Jiaxin Kang ${ }^{2, *}$, Zhuang Yao, ${ }^{3, *}$, Youli Jian ${ }^{4}$, Yudong Jing ${ }^{4}$, Yuanzhao \\ $H^{2}{ }^{2}$, Mei Yang ${ }^{4}$ and Jianwei Sun ${ }^{2}$ \\ ${ }^{1}$ College of Horticulture, Qingdao Agricultural University, Qingdao 266109, China \\ ${ }^{2}$ Guangdong Provincial Key Laboratory of Protein Function and Regulation in Agricultural Organisms, College of Life Sciences, \\ South China Agricultural University, Guangzhou, Guangdong 510642, China \\ ${ }^{3}$ Institute of Agriculture and Life Sciences, Gyeongsang National University, Jinju, 52828, Korea \\ ${ }^{4}$ Key Laboratory of Molecular and Developmental Biology, Institute of Genetics and Developmental Biology, Chinese Academy \\ of Sciences, Beijing, 100101, China \\ *These authors have contributed equally to this work \\ Correspondence to: Mei Yang, email: athensmei@gmail.com \\ Jianwei Sun, email: jwsun@scau.edu.cn \\ Keywords: nhr-14; DNA damage; apoptosis \\ Received: September 28, $2017 \quad$ Accepted: December 05, $2017 \quad$ Published: January 03, 2018 \\ Copyright: Shen et al. This is an open-access article distributed under the terms of the Creative Commons Attribution License 3.0 \\ (CC BY 3.0), which permits unrestricted use, distribution, and reproduction in any medium, provided the original author and source \\ are credited.
}

\section{ABSTRACT}

Nuclear hormone receptor is involved in transcription regulation and many important cellular processes including development and metabolism. However, the role of nuclear hormone receptor in DNA damage-induced apoptosis remains elusive. Here we reported that RNAi of $n h r-14$, which was thought to be an estrogenic hormone receptor in Caenorhabditis elegans, inhibited DNA damage-induced apoptosis in prmt5(gk357), a C. elegans homolog of mammalian type II arginine methyltransferase PRMT5, after ionizing radiation. Deletion of $n h r-14$ led to decreased DNA damageinduced germline apoptosis, but not in the physiological programmed cell death. We also demonstrate that $n h r-14$ is not a checkpoint gene and functions downstream of the checkpoint pathway. Moreover, we show that $n h r-14$ regulates egl-1 and ced13 transcription upon DNA damage. In addition, we provided evidence that NHR14 forms a complex with CEP-1/p53 and may function as a cofactor of CEP-1/p53. These findings indicate that NHR-14 might cooperate with CEP-1/p53 to regulate DNA damage-induced apoptosis, which reveals a novel role for nuclear hormone receptor in apoptosis.

\section{INTRODUCTION}

Programmed cell death (i.e., apoptosis) is one of the most important processes in the metazoans development. It plays the key roles in animal development and DNA damage repair. DNA damage-induced apoptosis is the cell death happening after severe DNA damage, which is associated with a number of human diseases including cancer. Caenorhabditis elegans has been used extensively to study the programmed cell death induced by DNA damage responses. In C. elegans, the p53 homolog CEP1 acts as a key effector to mediate germ cell apoptosis triggered by ionizing irradiation [1]. Although many factors have been reported to be involved in p53/cep-1 dependent apoptotic pathway, the details of this pathway is still not completely understood.

Nuclear hormone receptors (NHRs) comprise of a large family of transcription factors distinguished by a highly conserved DNA binding domain and a structurally conserved ligand-binding domain. There are 284 predicted 
NHR genes in C.elegans [2]. Nuclear hormone receptors have been shown to regulate important developmental process [3-6]. However, the role of NHR in programmed cell death has not been documented.

We previously demonstrated that prmt-5, the C. elegans homolog of mammalian type II arginine methyltransferase PRMT5, negatively regulates DNA damage-induced apoptosis [7]. prmt-5(gk357) deletion mutants have increased germline programmed cell death after DNA damage. Furthermore, genetic analyses indicated that prmt-5-mediated apoptosis depends on cep1/p53 and requires the core cell death pathway.

In the present study, we show that RNAi knockdown of $n h r-14 / \mathrm{HNF} 4$, which is thought to be an estrogen receptor in C. elegans[8], suppresses DNA damageinduced apoptosis in prmt-5(gk357) deletion mutant. Further, we show that $n h r-14 / \mathrm{HNF} 4$ is a new factor involved in the DNA damage-induced apoptosis and that $n h r-14$ is not a checkpoint gene and functions downstream of the checkpoint genes. Our study confirmed that NHR$14 / \mathrm{HNF} 4$, cooperates with CEP-1/p53 to regulate egl-1 (Bcl-2 homology region 3 domain containing gene) and ced-13 (Bcl-2 homology region 3 domain containing gene) expression and DNA damage-induced apoptosis, which may offer clinical target for cancer therapy.

\section{RESULTS}

\section{Inactivation of $\boldsymbol{n h r}$-14/HNF4 inhibits DNA damage-induced apoptosis}

To examine whether nuclear hormone receptor is directly involved in the regulation of DNA damageinduced apoptosis, we performed RNAi screen in the background of prmt-5(gk357). We found that knockdown of $n h r-14 / \mathrm{HNF} 4$ RNAi reduced the DNA damage-induced programmed cell death in prmt-5 (gk357) (Figure 1A) after ionizing irradiation.

C. elegans $n h r-14$ gene is defined by the open reading frame T01B10.4 located on the linkage group $\mathrm{X}$, which encodes a protein of 435 amino acids. nhr14(tm1473)contains a deletion of 409bp in the third exon and intron resulting in an early stop of NHR-14 translation [8].

In order to test the function of $n h r-14 / \mathrm{HNF} 4$ in DNA damage-induced apoptosis, we used $n h r-14$ (tm 1473) deletion mutant to analyze the germ cell apoptosis after ionizing irradiation, we found that $n h r-14(t m 1473)$ can inhibit DNA damage-induced apoptosis in prmt$5(\mathrm{gk357})$ at different gamma-irradiation dose (Figure 1B) and different time (Figure 1C), suggesting that nhr14 functions downstream of prmt-5 and regulates DNA damage-induced programmed cell death.

To further determine whether $n h r-14 / \mathrm{HNF} 4$ is a new factor that involved in the DNA damage induced cell apoptosis, we performed epistasis analysis using several well-defined cell survival molecules including
AKT-1/AKT, ABL-1/ABL and CED-9/BCL2. Previous studies have demonstrated that loss function mutation of C.elegans akt-1(ok525) exhibits dramatic increased programmed cell death after gamma-irradiation [9] and that mutation of $a b l-1 /$ abl1 induces more germline apoptosis than wildtype. Furthermore, it has been shown that loss function of ced-9, a BCL-2 homolog in C.elegans [10], activates CED-3 to induce programmed cell death $[11,12]$ and that ced-9(n1653) mutant exhibits more apoptotic cells upon DNA damage treatment. Our epistasis analysis revealed that $n h r-14 / \mathrm{HNF} 4$ deletion abrogated DNA damage-induced apoptosis in in akt1(ok525) (Figure 2A) and decreased apoptosis in ced9(n1653) background (Figure 2D). Knockdown of $n h r-14$ I HNF4 led to dramatically decreased germline apoptosis in abl-1(ok171) mutants (Figure 2B).

In addition, because $b r c-1$ is the BRCA1 homolog in C.elegans and functions in DNA double strand break (DSB) repair $[13,14]$ after ionizing gamma-irradiation, mutation of $b r c-1 / \mathrm{BRCA} 1$ resulted in failing to repair the double strand break and induce apoptosis. We also found that brc-1(tm1145);nhr-14(tm1473) double mutant exhibited dramatic decreased apoptosis compared to brc1(tm1145) alone after DNA damage (Figure 2C). Taken together, these findings indicate that $n h r-14 / \mathrm{HNF} 4$ is a key regulator of DNA damage-induced programmed cell death.

\section{nhr-14/HNF4 doesn't affect physiological programmed cell death}

Since $n h r-14$ (tm 1473) showed less apoptosis upon gamma-irradiation, we next investigated underlying cellular mechanism. We performed the time lapse phenotype analysis and found that there was no germline development defect and nhr-14(tm1473) showed the same apoptosis number as N2 at any time. These data indicate the decreased programmed cell death in $n h r-14$ (tm 1473) is neither due to germline development nor the delayed cell death. We further examined whether $n h r-14$ affects the physiological programmed cell death in embryo. Figure $3 \mathrm{~A}$ shows that there was no difference in the number of cell apoptosis in embryo between N2 and $n h r-14(t m 1473)$. ced-1(e1735) [15] and vps-18(tm1125) [16] has been reported to affect cell corpse clearance, we also found no difference in the number of cell apoptosis in germline between wild type and nhr-14(tm1473) mutant in the background of ced-1(e1735) [16] and vps-18(tm1125) [17] (Figure 3B, 3C). These results indicate that $n h r-14 / \mathrm{HNF} 4$ only affects the DNA damage-induced apoptosis, but not the physiological programmed cell death.

\section{nhr-14/HNF4 functions downstream of the checkpoint pathway}

Previous studies demonstrated that the checkpoint signaling pathways are activated upon DNA damage and play the critical role in repairing the damaged DNA or 
inducing programmed cell death [17, 18]. Mutations in checkpoint genes can restrain both DNA damage-induced cell cycle arrest and apoptosis upon gamma-irradiation in C. elegans [17]. To determine whether $n h r-14 / \mathrm{HNF} 4$ is a checkpoint gene, we first assessed the sensitivity of $n h r-14$ (tm 1473) mutant to gamma-irradiation using radiation sensitivity assay. We found that the survival rate of $n h r-14$ (tm 1473) progeny was comparable to that of wild-type animals, but was much higher than that of checkpoint gene mutant hus-1(op244) and clk-2(mn159) (Table 1). In addition, $n h r-14(t m 1473)$ worms displayed similar cell cycle arrest in germline mitotic region to that in wild type following irradiation-treatment (Figure 4A). We further made hus-1(op244); nhr-14(tm1473) and clk2(mn159);nhr-14(tm1473) double mutants, and found that these double mutants exhibited the same phenotype as the check point mutants (Figure 4B). Therefore, $n h r-14 / \mathrm{HNF} 4$ is not a checkpoint gene and may function downstream of the checkpoint pathway.

To determine whether $n h r-14 / \mathrm{HNF} 4$ is involved in DNA repair, we irradiated worms containing the hus$1: \because g f p$ transgene in $n h r-14(t m 1473)$ background. We found that relocalization of HUS-1::GFP was independent of $n h r-14 / \mathrm{HNF} 4$ (Supplementary Figure 1A), and the
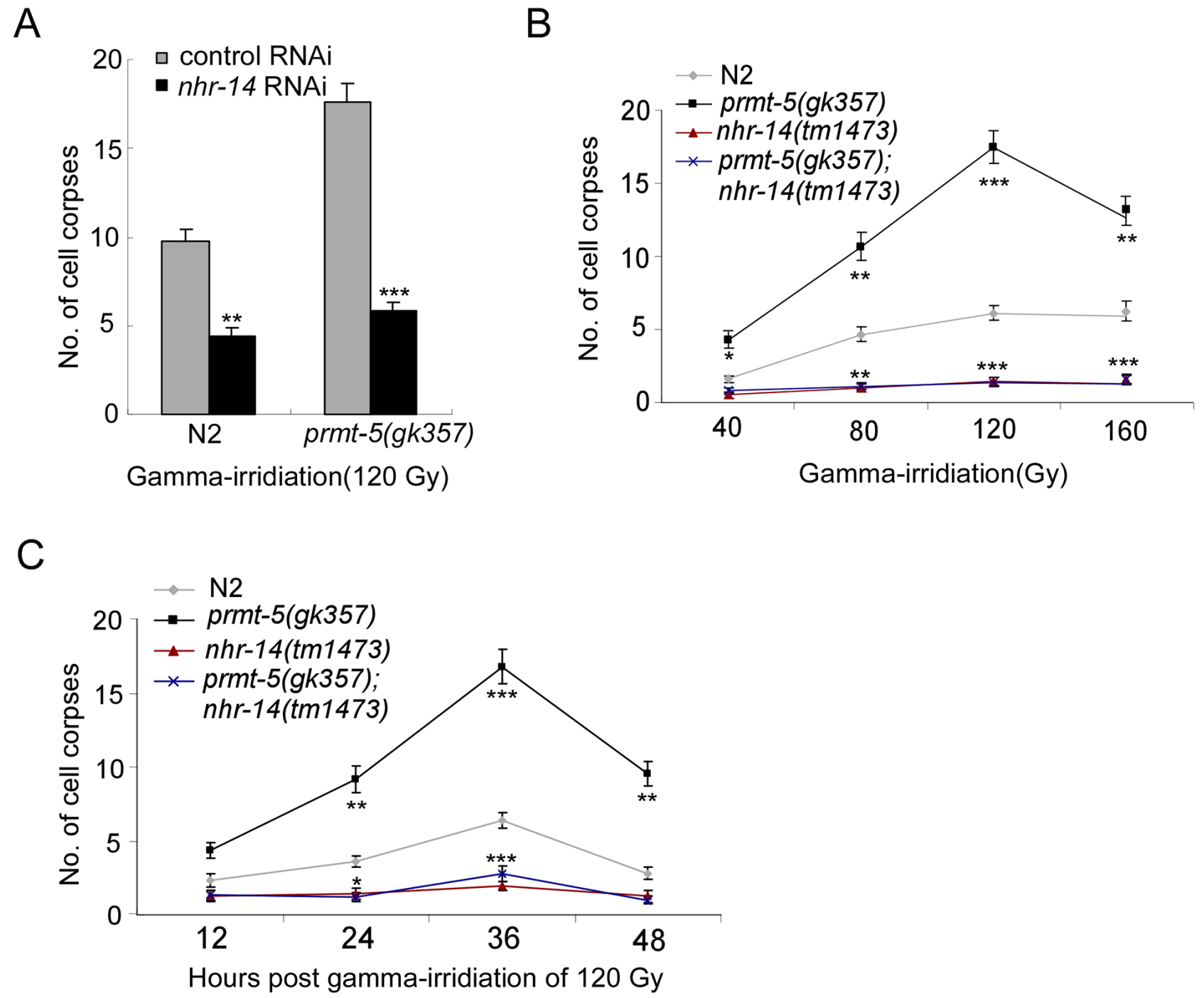

Figure 1: Inactivation of $\mathrm{nhr-14/HNF4}$ inhibits DNA damage-induced programmed cell death in prmt-5(gk357). (A) Quantitative analysis of germ cell apoptosis in control RNAi- and $n h r-14$ RNAi-treated N2 and prmt-5(gk357) animals. N2 and prmt5 ( $g k 357)$ were fed with control RNAi and $n h r-14$ RNAi and then (L4) was irradiated. After 36 hours of gamma-irradiation, germ cell apoptosis from one gonad arm of each animal were scored from at least 20 animals. Error bars represent standard error of the mean (SEM). ${ }^{* *}$ and ${ }^{* * *}$ indicate $\mathrm{p}<0.01$ and 0.001 , respectively. (B) Quantitative analysis germ cell apoptosis induced by gamma-irradiation in N2, $n h r-$ 14(tm 1473), prmt-5(gk357) and prmt-5(gk357); $n h r-14(t m 1473)$. Germ cell apoptosis from one gonad arm of each animal were scored after $36 \mathrm{~h}$ of irradiation at indicated doses. At least 20 worms were scored at each radiation dose or time point. Error bars represent standard error of the mean (SEM). (C) Quantitative analysis germ cell apoptosis at indicated time points after irradiation (120 Gy) in N2, nhr-14(tm1473), prmt-5(gk357) and prmt-5(gk357); nhr-14(tm1473) animals. 
number of foci in $n h r-14(t m 1473)$ was the same as wild type N2(Supplementary Figure 1B). nhr-14(tm1473) is defective in irradiation induced apoptosis but is wild type for irradiation induced cell cycle arrest (Figure 4) and DNA repair. These findings suggest that $n h r-14 / \mathrm{HNF} 4$ is not involved in DNA repair and acts downstream of the checkpoint genes.

\section{NHR-14/HNF4 cooperates with CEP-1/p53 to regulate egl-1 and ced-13 transcription upon DNA damage}

To investigate how $n h r-14 / \mathrm{HNF} 4$ regulates DNA damage-induced programmed cell death, we first examined the expression level of apoptotic initiator gene egl-1 and ced-13 in N2 and nhr-14(tm1473) worms. We irradiated $\mathrm{N} 2$ and $n h r-14$ (tm 1473) young adult worms at a dose of 120 Gy and performed RT-qPCR experiment, our results show that gamma-irradiation induced egl-1 and ced-13 levels were significantly reduced in $n h r-14(t m 1473)$. In N2 worms, egl-1 level was increased by 20 folds, however, in $n h r-14$ (tm 1473) egl-1 expression only increased 8 folds after DNA damage (Figure 5A). ced-13 expression level was induced more than 5 fold in N2 worms upon gammairradiation, but only about 3 fold in $n h r-14(t m 1473)$ worms (Figure 5B). These results suggest that $n h r-14 / \mathrm{HNF} 4$ regulates DNA damage-induced egl-1 and ced -13 .

Previous studies demonstrated that CEP-1/p53 is a key transcription factor of egl-1 and ced-13 [18, 19]. Because $n h r-14 / \mathrm{HNF} 4$ regulates egl-1 and ced-13 at mRNA level, we hypothesized that NHR-14/HNF4 could be a cofactor of CEP-1/p53. To this end, we first examined if NHR-14/HNF4 forms a complex with CEP-1/p53. Flagtagged NHR-14 and Myc-CEP-1 were co-transfected into $293 \mathrm{~T}$ cells, and then CoIP was performed. Figure 5C shows that Myc-CEP-1/p53 was co-immunoprecipitated with Flag-NHR-14, suggesting that these two proteins interact each other in mammalian cells. To investigate if NHR-14/HNF4 directly binds to CEP-1/p53, we performed in vitro GST-pull down assay. We found that
A

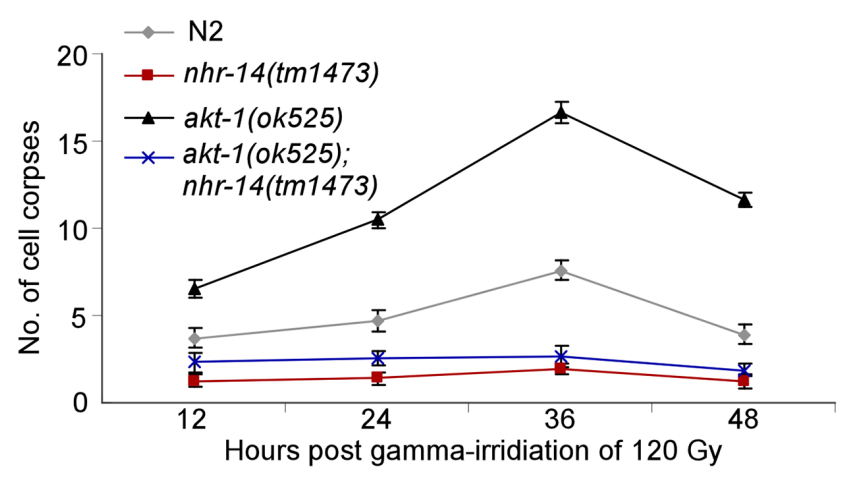

C

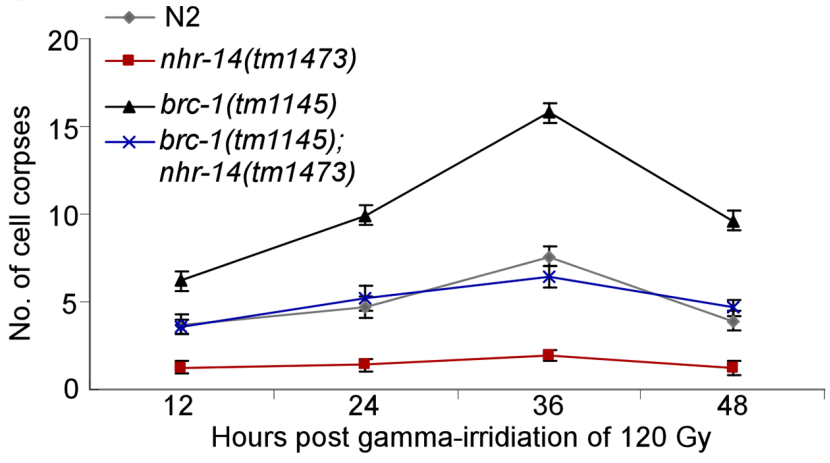

B
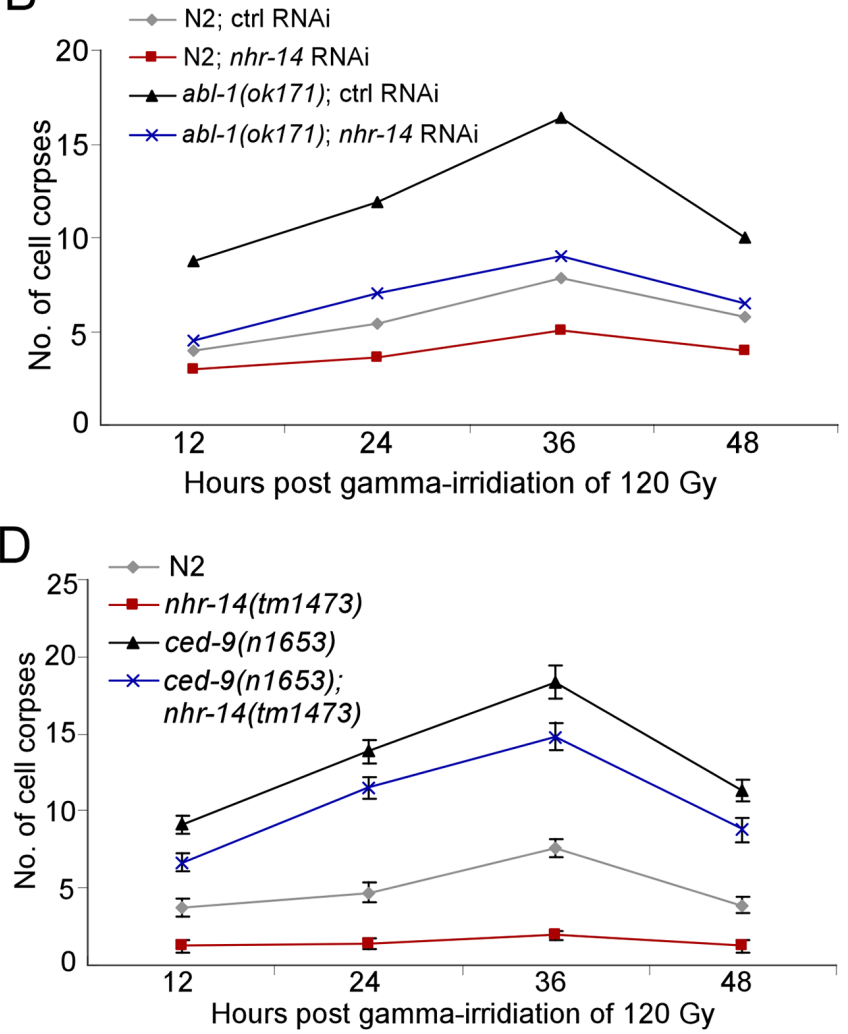

Figure 2: Epistasis analysis indicates nhr-14/HNF4 mediating DNA damage-induced apoptosis. (A) Quantitative analysis germ cell apoptosis induced by gamma-irradiation in N2, nhr-14(tm1473), akt-1(ok525) and akt-1(ok525); nhr-14(tm1473). Young adult animals were irradiated with gamma-ray $(120 \mathrm{~Gy})$ and analyzed at indicated time points after irradiation. Error bars represent standard error of the mean (SEM). (B) Quantitative analysis germ cell apoptosis in control RNAi and nhr-14 RNAi-treated N2 and abl-1(ok171) animals. N2 and abl-1(ok171) were fed with control RNAi and nhr-14 RNAi and then (L4) was irradiated at $120 \mathrm{~Gy}$. After 36 hours of gamma-irradiation, germ cell apoptosis from one gonad arm of each animal were scored from at least 20 animals. Error bars represent standard error of the mean (SEM). (C) Quantitative analysis of germ cell apoptosis induced by gamma-irradiation in N2, $n \mathrm{hr}-14$ (tm 1473), brc-1(tm 1145) and brc-1 (tm 1145); nhr-14(tm 1473) animals. (D) Quantitative analysis germ cell apoptosis induced by gamma-irradiation in $\mathrm{N} 2, n h r-14(t m 1473)$, ced-9(n1653) and ced-9(n1653); nhr-14(tm1473) animals. 
GST-CEP-1 fusion protein, but not GST, pulled-down ${ }^{[35 \mathrm{~S}]}$ methionine labeled NHR-14 (Figure 5D). These data suggests that NHR-14/HNF4 and CEP-1/p53 might directly interact each other.

We next investigated whether $n h r-14$ /HNF4 regulates DNA damage induced programmed cell death through cep-1/p53. As GLD-1 is a translational repressor of cep-1/p53 via directly binding to the 3'UTR of cep-1/p53 mRNA [20], gld-1(op236) loss of function mutant expresses higher level CEP-1/p53 in C.elegans. We found that egl-1 and ced-13 mRNA level were much higher in gld-1(op236) mutant than N2 worms after gamma-irradiation (Figure 5A, 5B). We also demonstrated that up-regulated CEP-1/p53 in gld-1(op236) could rescue DNA damage-induced egl1 and ced-13 expression and apoptosis (Figure 5A, 5B and $5 \mathrm{E}$ ).
In conclusion, our data suggest that $n h r-14 / \mathrm{HNF} 4$ functions as a cofactor of cep-1/p53 and regulates DNA damage-induced programmed cell death through CEP-1/ p53 (Figure 5F).

\section{DISCUSSION}

DNA damage-induced programmed cell death is associated with various human malignancies and identification of regulators in DNA damage-induced apoptosis pathway is critical for intervention of these diseases. C.elegans has been shown to be an excellent model to study DNA damage-induced programmed cell death. And thus it is very helpful for us to understanding the mechanism of carcinogenesis by studying the regulation of DNA damage-induced apoptosis in C.elegans germline.
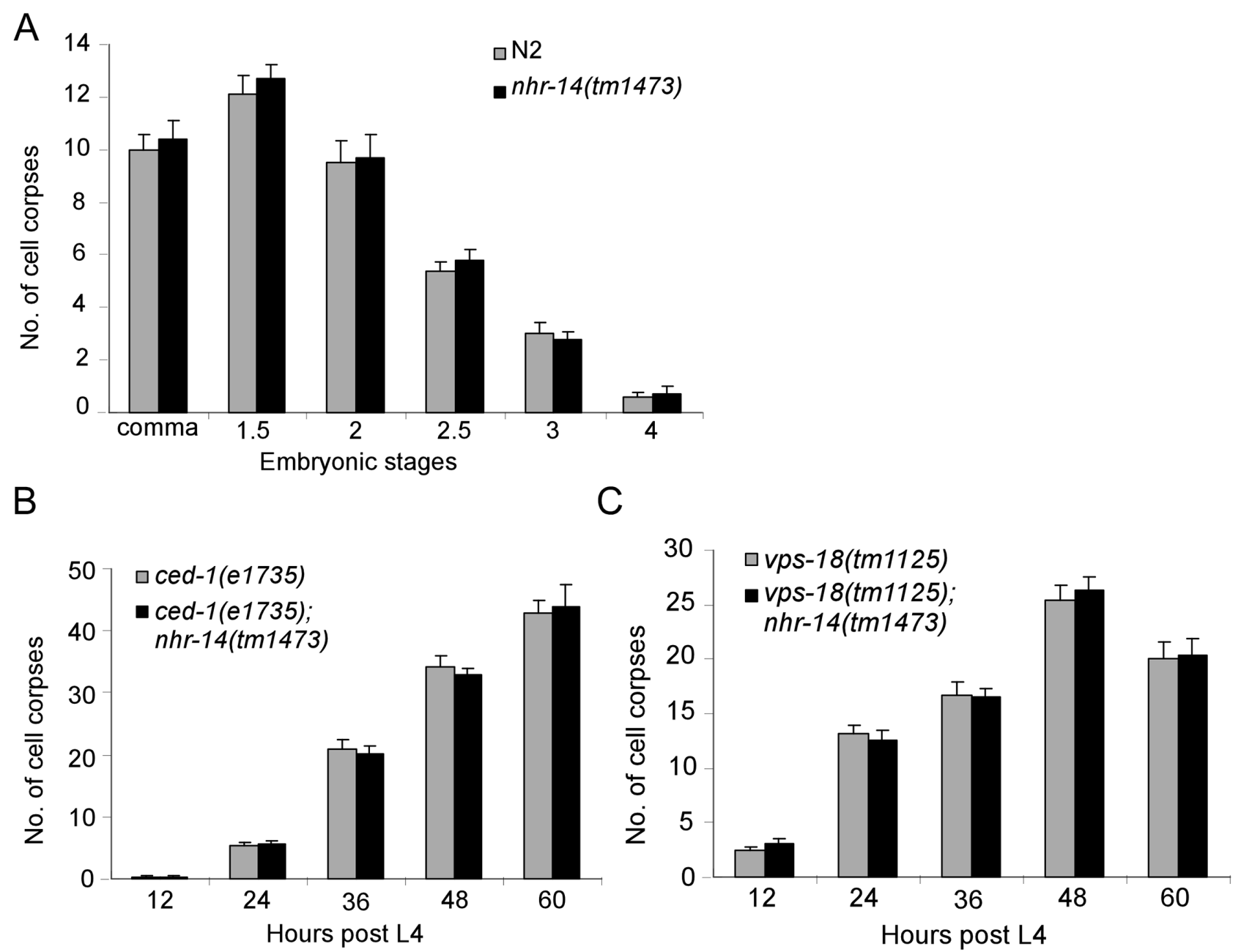

Figure 3: nhr-14 doesn't affect the physiological programmed cell death. (A) Quantification of embryo cell apoptosis in N2 and $n h r-14(t m 1473)$ mutants. (B) Quantification of germline cell apoptosis in ced-1(e1375) and ced-1(e1375); nhr-14(tm1473)animals at indicated time points post L4. (C) Quantification of germline cell apoptosis in vps-18(tm1125) and vps-18(tm1125); nhr-14(tm1473) animals at indicated time points post L4. 
Table 1: $n h r-14$ doesn't affect the survival of progeny after gamma-irradiation treatment the survival of nhr-14(tm 1473) mutant progeny is not sensitive to irradiation

\begin{tabular}{lcccccc}
\hline irradiation & \multicolumn{7}{c}{ Survival(\%) } \\
\hline Dose(Gy) & $N 2$ & $n h r-14(t m 1473)$ & $h u s-1($ op 244$)$ & $h u s-1 ; n h r-14$ & $c l k-2(m n 159)$ & $c l k-2 ; n h r-14$ \\
0 & $\mathbf{1 0 0 . 0} \pm \mathbf{0}$ & $\mathbf{1 0 0 . 0} \pm \mathbf{0}$ & $\mathbf{9 7 . 6} \pm \mathbf{0 . 1}$ & $\mathbf{9 8 . 3} \pm \mathbf{0 . 8}$ & $\mathbf{9 8 . 0} \pm \mathbf{0 . 7}$ & $\mathbf{9 6 . 0} \pm \mathbf{1 . 5}$ \\
40 & $\mathbf{8 6 . 3} \pm \mathbf{1 . 2}$ & $\mathbf{8 6 . 1} \pm \mathbf{1 . 8}$ & $\mathbf{3 7 . 7} \pm \mathbf{1 . 1}$ & $\mathbf{3 9 . 6} \pm \mathbf{2 . 0}$ & $\mathbf{2 9 . 1} \pm \mathbf{2 . 6}$ & $\mathbf{2 8 . 9} \pm \mathbf{1 . 7}$ \\
80 & $\mathbf{7 6 . 7} \pm \mathbf{2 . 4}$ & $\mathbf{7 6 . 3} \pm \mathbf{1 . 8}$ & $\mathbf{1 8 . 7} \pm \mathbf{1 . 3}$ & $\mathbf{1 8 . 0} \pm \mathbf{1 . 2}$ & $\mathbf{9 . 4} \pm \mathbf{0 . 5}$ & $\mathbf{1 1 . 1} \pm \mathbf{0 . 8}$ \\
$\mathbf{1 2 0}$ & $\mathbf{6 4 . 5} \pm \mathbf{1 . 6}$ & $\mathbf{6 2 . 5} \pm \mathbf{1 . 4}$ & $\mathbf{3 . 2} \pm \mathbf{0 . 5}$ & $\mathbf{3 . 5} \pm \mathbf{0 . 7}$ & $\mathbf{2 . 8} \pm \mathbf{1 . 0}$ & $\mathbf{2 . 8} \pm \mathbf{1 . 0}$ \\
\hline
\end{tabular}

P53 is a key tumor suppressor and its mutations were detected in more than $50 \%$ of human cancers. In C.elegans, the $\mathrm{p} 53$ homolog CEP-1 acts as a key effector to mediate germ cell apoptosis triggered by ionizing irradiation [21]. Identification of new co-factors of CEP$1 / \mathrm{p} 53$ in C.elegans may offer critical targets for cancer intervention.

In response to DNA damage stimuli, the checkpoint genes will sense the signals and induce cell cycle arrest or programmed cell death. Simultaneously, CEP-1/p53 is activated and subsequently induces up-regulation of BH3 genes egl-1 and ced-13. Mutation of the checkpoint genes block the transfer of DNA damage signals and reduce DNA damage-induced apoptosis. Nuclear hormone receptor family is a key to many important cellular processes, but the role of NHR family in DNA damageinduced programmed cell death remains elusive. Previous study showed that NHR-14/HNF4, which was thought to be an estrogenic hormone receptor [8], was involved in the immune response processes via regulation of vitellogenin expression [22]. In present report, we identified $n h r-14 /$ HNF4 as an important member of NHR in regulation of DNA damage-induced apoptosis. Moreover, we showed that $n h r-14 / \mathrm{HNF} 4$ is primarily involved in regulation of the DNA damage-induced apoptosis, but not the physiological programmed cell death (Figure 3).

Mechanically, our experiment revealed that $n h r$ 14 /HNF4 deletion decreases DNA damage-induced upregulation of egl-1 and ced-13. More significantly, we showed that NHR-14/HNF4 might interacts with CEP-1/ p53 and functions as a cofactor of CEP-1/p53. In addition, nhr-14(tm1473) mutant dramatically reduces CEP-1/p53mediated DNA damage-induced apoptosis. Therefore, our study first reported a nuclear hormone receptor NHR-14/ HNF4 that is involved in DNA damage-induced apoptosis. Identification of NHR-14/HNF4 interaction with CEP-1/ p53 to control DNA damage-induced egl-1 and ced-13 could provide new targets for cancer intervention.
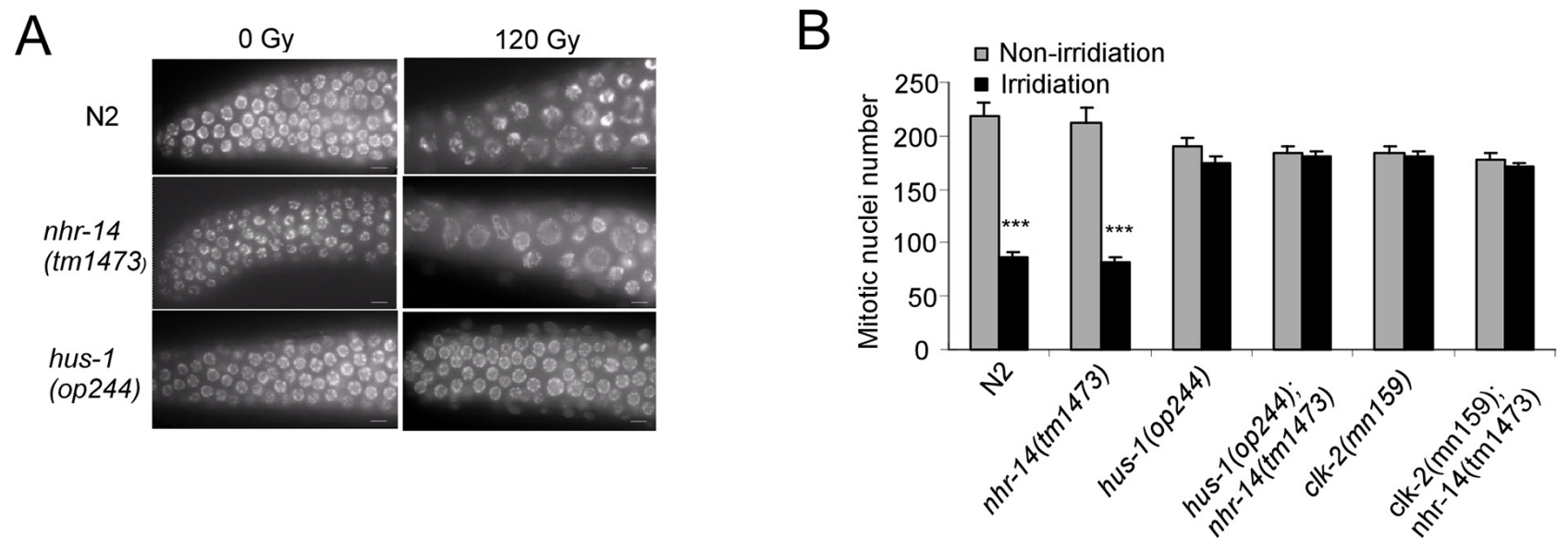

Figure 4: nhr-14 is not a checkpoint gene and does not affect the cell cycle arrest after DNA damage. (A) Representative image of DAPI staining of the germline mitotic region in N2, $n h r-14$ (tm 1473) and hus-1(op234) worms. Young adult worms were treated with gamma-irradiation at $120 \mathrm{~Gy}$. After 36 hours of irradiation, germline was dissected and stained with DAPI. Bars, $5 \mu \mathrm{m}$. (B) Quantification of germline mitotic nuclear number after gamma-irradiation in N2, nhr-14(tm1473), hus-1(op244), hus-1(op234);nhr-14(tm1473), clk2(mn159), clk-2(mn159);nhr-14 (tm1473) worms. The gray and black bars represent nuclei number in mitotic region in control and gamma irradiation-treated worm germline, respectively. ${ }^{* * *}$ indicate $\mathrm{p}<0.001$. 
A

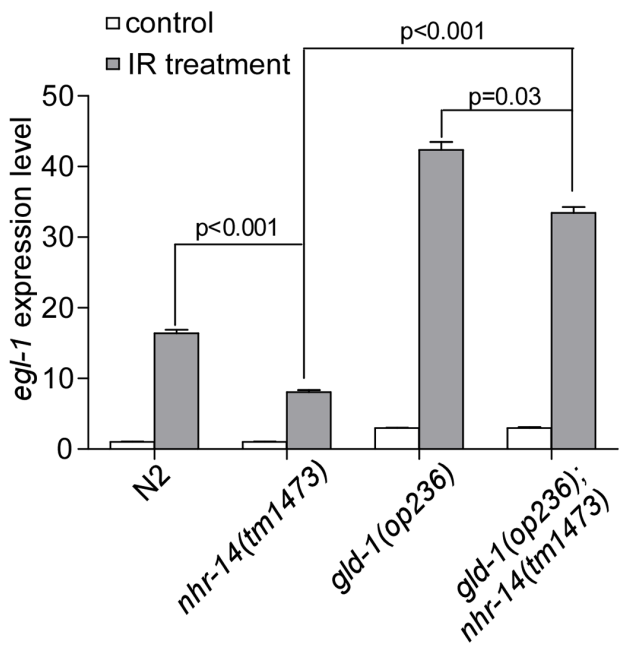

C

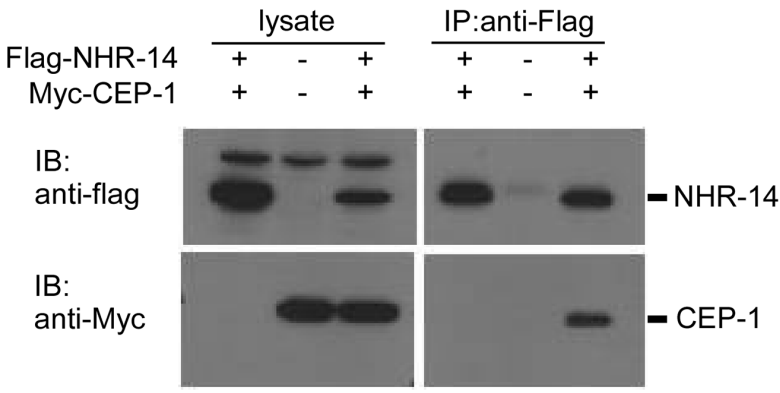

$E$

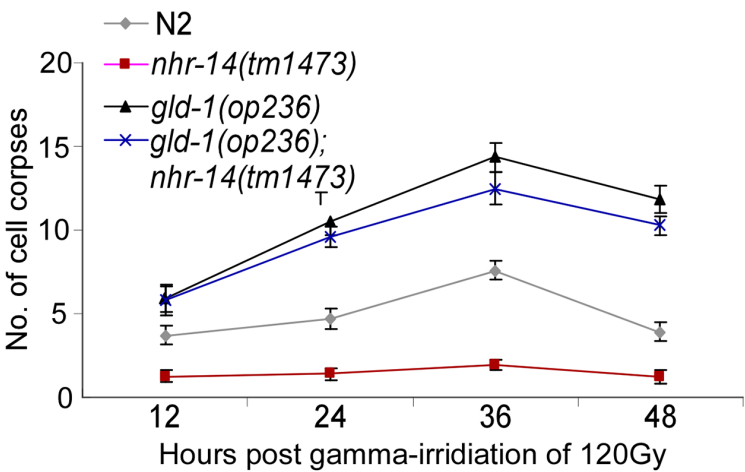

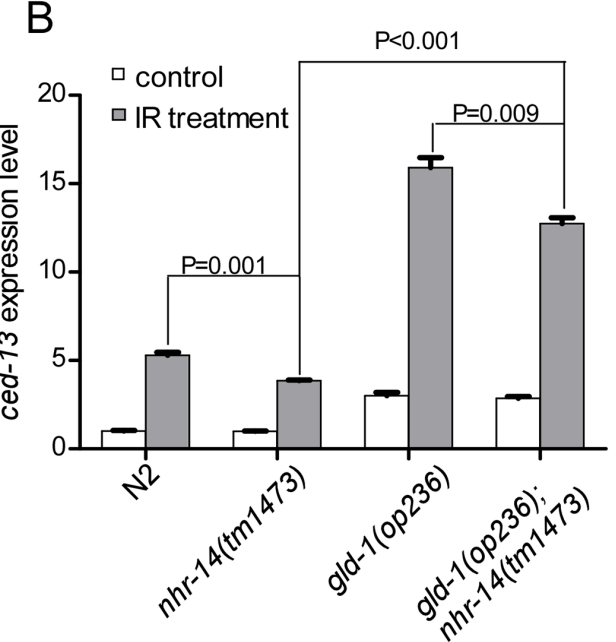

$\mathrm{D}$

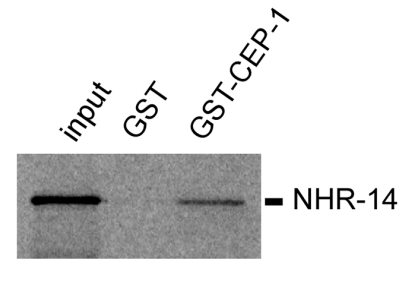

$\mathrm{F}$

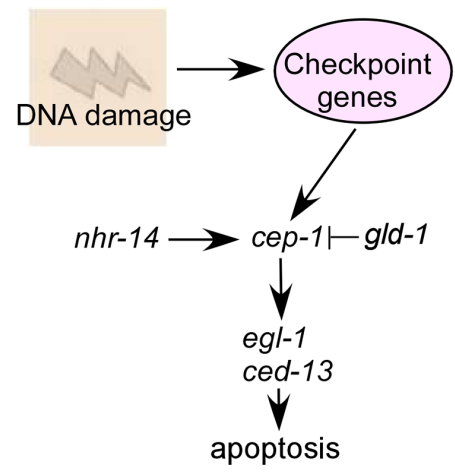

Figure 5: NHR-14 cooperates with CEP-1/p53 to regulate egl-1 and ced-13 expression. (A) Relative fold induction of egl-1 mRNA in N2, nhr-14(tm1473), gld-1(op236) and gld-1(op236);nhr-14(tm1473) after 24 hours of gamma-irradiation (120 Gy). egl-1 fold induction was averaged from three independent RT-qPCR analyses. (B) Relative fold induction of ced-13 mRNA in N2, nhr-14(tm 1473), gld-1(op236) and gld-1(op236);nhr-14(tm1473) following 24 hours of gamma-irradiation (120 Gy). egl-1 fold change was averaged from three independent RT-qPCR analyses. (C) NHR-14 interacts with CEP-1/p53 in mammalian cells. Flag-NHR-14 and Myc-CEP-1/ p53 were co-expressed in HEK293 cells and then immunoprecipitated (IP) using Flag antibody. The immunoprecipitated proteins were detected by immuno-blotting (IB) with Myc antibody. (D) NHR-14 and CEP-1/p53 directly interact in vitro. The full-length NHR-14 protein was in vitro translated and labeled with $\left.{ }^{35} \mathrm{~S}\right]$ methionine and incubated with GST or GST-CEP-1/p53 fusion proteins, which were immobilized on glutathione sepharose beads, for $2 \mathrm{~h}$. After extensive washes, the bound proteins were resolved by SDS-PAGE and detected by autoradiography (E) Quantitative analysis germ cell apoptosis induced by gamma-irradiation at indicated time points post L4 in N2, $n h r$-14(tm1473), gld-1(op236) and gld-1(op236);nhr-14(tm1473). Up-regulated CEP-1/p53 via loss function of gld-1(op236) rescued DNA damage-induced programmed cell death in $n h r-14(t m 1473)$ animals. (F) The genetic pathway for $n h r-14 /$ HNF4 to regulate DNA damageinduced apoptosis. NHR-14/HNF4 functions as a cofactor of CEP-1/p53 to regulate DNA damage induced apoptosis via controlling egl-1 and ced-13 expression. 


\section{MATERIALS AND METHODS}

\section{C. elegans strains and genetics}

The strains of $n h r-14(t m 1473), b r c-1(t m 1145)$ and $v p s-18(t m 1125)$ were provided by Dr. Shohei Mitani. prmt-5(gk357), cep-1(gk138), gld-1(op236), akt-1(ok525), abl-1(ok171), ced-9(n1653), hus-1(op244) and clk2 (mn159) strains were provided by C. elegans Genetic Center (CGC). Worms were cultured and maintained using standard procedures. The Bristol N2 strain was used as wildtype. Deletion strains were outcrossed with N2 strain for 6 times. Double mutants were constructed with standard protocol.

\section{Germ cell apoptosis assay}

Synchronized young adult animals were irradiated with gamma-Ray (120Gy), which was located in the Peking University Health Science Center. Irradiated animals were put back to culture at $20^{\circ} \mathrm{C}$ at different time points. Worms with normal germline morphology were scored for germline cell apoptosis with DIC Zeiss microscope.

\section{Radiation sensitivity assay}

N2 worms and $n h r-14(t m 1473)$ and hus-1(op244), hus-1(op244);nhr-14(tm1473), clk-2(mn159), clk2(mn159);nhr-14(tm1473) mutant worms were irradiated at the L4 stage as indicated. Eggs laid 8-24hr after irradiation (corresponding to pachytene-stage germ cells at the time of irradiation) were counted. Unhatched eggs surviving animals were counted for days 1 and 2 . The result represents the percent of survival of embryos of six different animals per strain.

\section{Mammalian cell culture, transfection and immunoprecipitation}

Human embryonic kidney (HEK293) cells were grown in Dulbecco's modified Eagle's medium (HyClone) supplemented with $10 \%$ fetal bovine serum (HyClone). The transfection was performed with $2.0 \mu \mathrm{g}$ of mammalian vectors expressing worm proteins with different tags (i.e., pCMV-myc-cep-1, pCMV-tag2B-nhr-14) using PEI reagent. After $36 \mathrm{~h}$ of transfection, cells were harvested and lysed in a buffer containing $50 \mathrm{mM}$ Tris $(\mathrm{pH} 8.0), 150 \mathrm{mM}$ $\mathrm{NaCl}, 0.5 \%$ sodium deoxycholate, $1 \%$ Triton $\mathrm{X}-100$, $1 \mathrm{mM}$ phenylmethylsulfonyl fluoride (PMSF). The lysate was incubated with anti-Flag antibody (M2)-conjugated agarose beads (Sigma) for more than $2 \mathrm{~h}$ at $4^{\circ} \mathrm{C}$. The beads were washed extensively in a buffer containing $50 \mathrm{mM}$ Tris ( $\mathrm{pH} 8.0), 150 \mathrm{mM} \mathrm{NaCl}, 1 \mathrm{mM}$ PMSF and 1\% NP40. Bound proteins were eluted and resolved on sodium dodecyl sulfate polyacrylamide gel electrophoresis (SDSPAGE) and detected with Western blot assay.

\section{Western blot assay}

Cells were scraped and lysed in lysis buffer on ice for $15 \mathrm{~min}, 15 \mu \mathrm{g}$ total proteins were loaded on SDSPAGE Gel as co-immunoprecipitation experiment input. The SDS-PAGE gel first run on $60 \mathrm{~V}$ for $30 \mathrm{~min}$ and then $120 \mathrm{~V}$ until the dye run out of the gel, then the protein was transferred to PVDF membrane. The membranes were blocked in 5\% nonfat dry milk in Tris-buffered saline, $0.05 \%$ Tween for 30 minutes at room temperature, and then incubated with primary antibodies for 2 4hours at $4^{\circ} \mathrm{C}$, followed by incubation with secondary antibody for 60 min at RT. The primary antibodies used in this study were as follows:

anti-Flag (Sigma, Cat\#:F3165) and anti-Myc (Sigma, Cat\#:HPA055893).

\section{GST pull-down assay}

For GST pull-down assay, purified GST or GSTCEP-1 fusion proteins were immobilized on glutathioneSepharose beads and incubated with ${ }^{[35 S]}$ methioninelabeled NHR- 14 at $4^{\circ} \mathrm{C}$ for more than $2 \mathrm{~h}$. The beads were washed extensively and bound proteins were eluted and separated on $12 \%$ SDS-PAGE and exposed to phosphoimager (Amersham) for autoradiography.

\section{RT-qPCR assay}

Total C.elegans RNA was extracted using TRIZOL method and cDNA was synthesized using iScript cDNA Synthesis Kit (Bio-Rad Laboratories). qPCR was performed in an iCycler thermocycler (Bio-Rad Laboratories) using iQ SYBR Green Supermix (Bio-Rad Laboratories). mRNA levels were quantified using iCycler software (Bio-Rad Laboratories) and were normalized to $t b g-1$. The primers used for RT-qPCR were as follows:

egl-1 q-PCR NS: 5'-gattcttctcaatttgccgacg-3'; egl-1 q-PCR CAS: 5'-tcatctgagcatcgaagtcatc-3'; ced-13 q-PCR NS: 5'-acggtgtttgagttgcaagc-3';

ced-13 q-PCR CAS: 5' -gtcgtacaagcgtgatggat-3; tbglq-PCR NS: 5'-cgtcatcagcctggtagaaca-3'; tbg-lq-PCR CAS: 5'-tgatgactgtccacgttgga-3'.

All experiments were analyzed in triplicates.

\section{ACKNOWLEDGMENTS AND FUNDING}

We thank C. elegans Genetic Center and Dr. Shohei Mitani for providing C.elegans deletion strains. We especially thank Dr. Chonglin Yang for helpful suggestion and reagents. We also thank Dr. Shengyu Yang for comments on the manuscript.

This work was supported by National Natural Science Foundation of China (31671448 and 81472264), Natural Science Foundation of Shandong Province (ZR 2015CL034) and Advanced Talents Foundation of Qingdao Agricultural University (663/1113307). 


\section{CONFLICTS OF INTEREST}

The authors declare no competing financial interests.

\section{REFERENCES}

1. Schumacher B, Hofmann K, Boulton S, Gartner A. The C. elegans homolog of the $\mathrm{p} 53$ tumor suppressor is required for DNA damage-induced apoptosis. Curr Biol. 2001; 11:172227. https://doi.org/10.1016/S0960-9822(01)00534-6.

2. Antebi A. Nuclear hormone receptors in C. elegans. WormBook. 2006; 1-13.

3. Magner DB, Wollam J, Shen Y, Hoppe C, Li D, Latza C, Rottiers V, Hutter H, Antebi A. The NHR-8 nuclear receptor regulates cholesterol and bile acid homeostasis in C. elegans. Cell Metab. 2013; 18:212-24. https://doi. org/10.1016/j.cmet.2013.07.007.

4. Verghese E, Schocken J, Jacob S, Wimer AM, Royce R, Nesmith JE, Baer GM, Clever S, McCain E, Lakowski B, Wightman B. The tailless ortholog nhr-67 functions in the development of the C. elegans ventral uterus. Dev Biol. 2011; 356:516-28. https://doi.org/10.1016/j. ydbio.2011.06.007.

5. Goudeau J, Bellemin S, Toselli-Mollereau E, Shamalnasab M, Chen Y, Aguilaniu H. Fatty acid desaturation links germ cell loss to longevity through NHR-80/HNF4 in C. elegans. PLoS Biol. 2011; 9:e1000599. https://doi.org/10.1371/ journal.pbio.1000599.

6. Liang B, Ferguson K, Kadyk L, Watts JL. The role of nuclear receptor NHR-64 in fat storage regulation in Caenorhabditis elegans. PLoS One. 2010; 5:e9869. https:// doi.org/10.1371/journal.pone.0009869.

7. Yang M, Sun J, Sun X, Shen Q, Gao Z, Yang C. Caenorhabditis elegans protein arginine methyltransferase PRMT-5 negatively regulates DNA damage-induced apoptosis. PLoS Genet. 2009; 5:e1000514. https://doi. org/10.1371/journal.pgen.1000514.

8. Mimoto A, Fujii M, Usami M, Shimamura M, Hirabayashi N, Kaneko T, Sasagawa N, Ishiura S. Identification of an estrogenic hormone receptor in Caenorhabditis elegans. Biochem Biophys Res Commun. 2007; 364:883-88. https:// doi.org/10.1016/j.bbrc.2007.10.089.

9. Quevedo C, Kaplan DR, Derry WB. AKT-1 regulates DNA-damage-induced germline apoptosis in C. elegans. Curr Biol. 2007; 17:286-92. https://doi.org/10.1016/j. cub.2006.12.038.

10. Hengartner MO, Horvitz HR. C. elegans cell survival gene ced-9 encodes a functional homolog of the mammalian proto-oncogene bcl-2. Cell. 1994; 76:665-76. https://doi. org/10.1016/0092-8674(94)90506-1.

11. Seshagiri S, Miller LK. Caenorhabditis elegans CED-4 stimulates CED-3 processing and CED-3-induced apoptosis. Curr Biol. 1997; 7:455-60. https://doi.org/10.1016/ S0960-9822(06)00216-8.
12. Spector MS, Desnoyers S, Hoeppner DJ, Hengartner MO. Interaction between the $\mathrm{C}$. elegans cell-death regulators CED-9 and CED-4. Nature. 1997; 385:653-56. https://doi. org/10.1038/385653a0.

13. Adamo A, Montemauri P, Silva N, Ward JD, Boulton SJ, La Volpe A. BRC-1 acts in the inter-sister pathway of meiotic double-strand break repair. EMBO Rep. 2008; 9:287-92. https://doi.org/10.1038/sj.embor.7401167.

14. Boulton SJ, Martin JS, Polanowska J, Hill DE, Gartner A, Vidal M. BRCA1/BARD1 orthologs required for DNA repair in Caenorhabditis elegans. Curr Biol. 2004; 14:3339. https://doi.org/10.1016/j.cub.2003.11.029.

15. Zhou Z, Hartwieg E, Horvitz HR. CED-1 is a transmembrane receptor that mediates cell corpse engulfment in C. elegans. Cell. 2001; 104:43-56. https:// doi.org/10.1016/S0092-8674(01)00190-8.

16. Xiao H, Chen D, Fang Z, Xu J, Sun X, Song S, Liu J, Yang C. Lysosome biogenesis mediated by vps-18 affects apoptotic cell degradation in Caenorhabditis elegans. Mol Biol Cell. 2009; 20:21-32. https://doi.org/10.1091/mbc. E08-04-0441.

17. Gartner A, Milstein S, Ahmed S, Hodgkin J, Hengartner MO. A conserved checkpoint pathway mediates DNA damage-induced apoptosis and cell cycle arrest in C. elegans. Mol Cell. 2000; 5:435-43. https://doi.org/10.1016/ S1097-2765(00)80438-4.

18. Hofmann ER, Milstein S, Boulton SJ, Ye M, Hofmann JJ, Stergiou L, Gartner A, Vidal M, Hengartner MO. Caenorhabditis elegans HUS-1 is a DNA damage checkpoint protein required for genome stability and EGL1-mediated apoptosis. Curr Biol. 2002; 12:1908-18. https:// doi.org/10.1016/S0960-9822(02)01262-9.

19. Schumacher B, Schertel C, Wittenburg N, Tuck S, Mitani S, Gartner A, Conradt B, Shaham S. C. elegans ced-13 can promote apoptosis and is induced in response to DNA damage. Cell Death Differ. 2005; 12:153-61. https://doi. org/10.1038/sj.cdd.4401539.

20. Schumacher B, Hanazawa M, Lee MH, Nayak S, Volkmann K, Hofmann ER, Hengartner M, Schedl T, Gartner A. Translational repression of C. elegans p53 by GLD-1 regulates DNA damage-induced apoptosis. Cell. 2005; 120:357-68. https://doi.org/10.1016/j.cell.2004.12.009. Erratum in: Cell. 2005; 122:145. Hofmann, Randall [corrected to Hofmann, E Randal].

21. Han Z, Riefler GM, Saam JR, Mango SE, Schumacher JM. The C. elegans Tousled-like kinase contributes to chromosome segregation as a substrate and regulator of the Aurora B kinase. Curr Biol. 2005; 15:894-904. https://doi. org/10.1016/j.cub.2005.04.019.

22. Fischer M, Regitz C, Kahl M, Werthebach M, Boll M, Wenzel U. Phytoestrogens genistein and daidzein affect immunity in the nematode Caenorhabditis elegans via alterations of vitellogenin expression. Mol Nutr Food Res. 2012; 56:957-65. https://doi.org/10.1002/mnfr.201200006. 